XV.

\title{
Sclerosirende Myositis der Zungenwurzel, einen malignen Tumor vortïuschend.
}

Von

Dr. O. Kappeler, Chefarzt in Münsterlingen.

(Mit einer mikroskopischen Untersuchung der exstirpirten Muskelstücke von Prof. Dr. M. Roth in Basel.)

Vor einigen Monaten kam mir ein höchst merkwürdiger Fall zur Beobachtung, wo in verhältnissmässig kurzer Zeit ein hühnereigrosser Tumor zwischen Unterkiefer und Zungenbein sich bildete, der vermöge seines raschen Wachsthums und seiner harten Consistenz als bösartige Neubildung imponirte, sich dann aber bei der Excision als sclerosirende Myositis mehrerer Zungen- und Zungenbeinmuskeln erwies. Vergeblich sah ich mich in der Literatur nach einem gleichen oder ähnlichen Krankheitsbilde um, wie mein Fall es bot. In den Handbüchern und speciell bei Otto Weber ${ }^{1}$ ) ist zwar eine Beobachtung Lambl's ${ }^{2}$ ) von einer am Halse prominirenden Geschwulst bei einem 11 jährigen Mädchen als Bindegewebswucherung der Zungenwurzel aufgefiihrt, allein es handelte sich bier nach der anatomischen Beschreibung offenbar um eine bösartige Neubildung. Auch Vir chow ${ }^{3)}$ hält den L a mbl'schen Tumor für einen fibrösen Krebs. Und so wäre denn unsere Beobachtung die einzige ihrer Art und somit vielleicht dazu angethan, die Kenntnisse über die Zungengeschwiilste in Etwas zu erweitern. Ich lasse zuerst die Krankheitsgeschichte folgen und füge am Schlusse einige epikritische Bemer. kungen an:

Der 26 jährige Land wirth P. Friedrich, der am 6. September 1881 in das hiesige Spital eintrat, stammt ans gesunder Familie, der betagte Vater

1) Handbuch der Chirurgie von Billroth-Pitha.

2) Löschner und Iambl, Aus dem Franz-Joseph-Kinderspital in Prag. Bcobachtungen und Erfahrungen 11. s. w. I. Theil. Prag 1816\%.

3) Geschwülste. III. Bd. S. 99.

Deutscrhe Zeitsehrift f. Chirurgie. XVI. Ba. 
arbeitet noch rüstig, die Mutter, die in Folge eines Gelenkrheumatismus herzleidend sein soll, ist trotzdem noch arbeitsfähig. Die 3 Geschwister des Patienten sollen sämmtlich vollkommen gesund sein. Der Kranke selbst war in seinen Knabenjahren, besonders im 12. Lebensjahre etwas anämisch und schwächlich, doch erholte er sich später wieder vollkommen und war vom 16. Jahre an auch nicht eine Stunde krank und arbeitsunfähig. Das Leiden, das ihnen hierher führte, soll vor 8 Wochen begonnen haben, vor dieser Zeit bemerkte er nicht das geringste davon. Damals (vor 8 Wochen) hatte er einige Tage Schluckbeschwerden, brennende Schmerzen beim Schlingen im vorderen Theil der Zunge. Diese Symptome gingen spontan wieder zurïck, und 2-3 Wochen später, also 5-6 Wochen vor dem Spitaleintritt, bildete sich unter dem Kinn eine Geschwulst, während zu gleicher Zeit dem Patienten die Zunge "wie angewachsen " erschien, das Sprechen undentlich und das Essen compacter Nahrung schwierig und schmerzhaft wurde. Pat. wandte sich hülfesuchend an einen Arzt, der das Leiden für einen Abscess gehalten zu haben scheint, denn er machte im Laufe der folgenden 2 Wochen 3 mal unterhalb des Kinns Incisionen, die jedoch jeweils nur etwas Blut entleerten. Die Geschwulst vergrösserte sich während dieser Zeit und die übrigen Beschwerden blieben unverändert bestehen. Ein zweiter Arzt, der ebenfalls berathen wurde, diagnosticirte eine Neubildung und instradirte den Kranken in ein auswärtiges Spital, woselbst er 5 Tage blieb. Hier wurde die Diagnose auf ${ }_{n}$ Speicheldrüsengeschwulst " gestellt und eine daselbst vorgenommene Incision links vom Frenulum soll 2-3 bohnengrosse, weisse, kalkige Körper zu Tage gefördert haben. Zu dieser Zeit noch gehörte die Geschwulst hauptsächlich der linken Seite an, die Zunge war besonders links am Mundboden fixirt und die Bewegungen derselben waren namentlich nach links hin gehemmt. Die Entfernung der Speichelsteine brachte keine Besserung, und der Kranke ging wieder nach Hause. In letzter Zeit entwickelte sich die Geschwulst auch nach rechtshin stärker, die Behinderung im Sprechen wurde noch grösser und die Schmerzen bei Kau- und Schlingbewegungen nahmen mehr und mehr zu.

Das Allgemeinbefinden hatte durch die Localerkrankung nicht im Entferntesten gelitten. Patient war bei der Anfnahme ins Spital von gesundem blühendem Aussehen, batte eine ausserordentlich kräftige Musculatur, gut entwickelte Knochen und fühlte sich mit Ausnahme der Sprech- und Schlingbeschwerden ganz wohl. Temperatur und Puls waren normal, und die Untersuchung der innern Organe, speciell der Lungen und des Herzens, förderte nichts Pathologisches zu Tage. Unterhalb des Kinns, den ganzen Kaum zwischen Kiefersymphyse und Zungenbein ausfüllend, sah und fühlte man eine mit der Haut nirgends verwachsene, rundliche, circa hühnereigrosse Geschwulst, die, völlig median gelegen, sich anf beiden Seiten bis zur Spitze des Cornu majus des Zungenbeins erstreckte, mit dem Unterkiefer im fester Verbindung war, in der Richtung von vorn nach hinten sich nicht verschieben liess, dagegen ziemlich ausgiebige seitliche Beweglichkeit zeigte. Zungenbein und Kehlkopf machten die seitlichen Bewegungen mit. Auch vom Boden der Mundhöhle alls, deren Schleimhaut normale Beschaffenheit zeigte, liess sich der Tumor genau abtasten und bis unter die Mitte der Zunge verfolgen. Von dem in der Mundhöhle ge- 
legenen Theil der Zunge war er ziemlich scharf abgegrenzt. Von oben wie von unten fühlte sich die Geschwulst hart elastisch an, zeigte nirgends weichere Partien und war auf starken Druck schmerzhaft. Die Dorsalfläche der Zunge zeigte in Bezug auf Grösse, Consistenz, Beschaffenheit der Schleimhaut und der Papillen durchans nichts Abnormes, nicht aber in Bezug anf Beweglichkeit, die nach allen Richtungen sehr eingeschränkt war. Sie konnte nach rechts und links bis auf die Zahnreihen translocirt werden, dagegen war das Aufstellen der Zungenspitze ganz unmöglich, und nach vorne konnte sie nur mit Anstrengung bis zum Lippensaum geschoben werden. Die Schlingbewegungen waren sehr gehindert und sehr schmerzhaft, die Sprache war undeutlich, pappig. Einzelne Buchstaben, wie $\mathrm{C}, \mathrm{Z}, \mathrm{W}, \mathrm{Q}$, konnte der Kranke überhaupt nicht ausprechen. Beide Tonsillen geschwollen, die linke mehr, als die rechte. Zu beiden Seiten des Tumors war je eine vergrösserte, bewegliche, mittelharte Lymphdrüse zu fühlen.

Es schien mir in hohem Grade wahrscheinlich, dass es sich um einen malignen Tumor handle. Schon das rasche Wachsthum der Geschwulst (die diese bezüglichen Angaben des Kranken lauteten sehr bestimmt) wies auf eine maligne Neubildung, und gestïtzt wurde diese Annahme durch die hart elastische Consistenz und die geringe Verschiebbarkeit, sowie durch deutliche Schwellung der benachbarten Lymphdrüsen. Als Ausgangspunkt der Neubildung betrachtete ich die Gland. sublinguales.

Am 8. September ging ich an die Exstirpation der Geschwulst. Ein medianer Längsschnitt vom Kiefer bis zur Schildknorpelincisur legte, nachdem die Zunge vorerst durch eine Fadenschlinge fixirt war, das Operationsgebiet frei. Um seitlich mehr Platz zu gewinnen, wurde nach Durchschneidung des Platysma der linke Digastricus an seiner Ansatzstelle vom Kiefer losgetrennt und zuriickgeschlagen. Bald lagen die Geschwulstmassen frei zu 'Tage, doch alsbald war auch zu erkennen, dass es sich keineswegs um eine Geschwulst im eigentlichen Sinn des Wortes handle, sondern um eine diffuse Infiltration von Zungen - und Kieferzungenbeinmuskeln. Da dieselbe, wie bald ersichtlich, noch keine allzugrosse Ausdehnung erfahren latte, und eine Totalexstirpation der erkrankten Partien möglich schien, wurde dieselbe ohne Verzug ins Werk gesetzt, und zwar musste zu diesem Behuf der grösste Theil der Geniohyodei, des Mylohyoideus und des Genioglossus entfernt werden. Es war nicht möglich bei dieser Excavation der Zunge von unten die Schleimhaut des Mundbodens unversehrt zu lassen, und es mussten mehrere grössere Aeste der Art. ligual. ligirt werden. Die gesunden Theile der Zunge liessen sich leicht und scharf durch den palpirenden Finger von den fast knorpelharten erkrankten Partien abgrenzen. Die mit der Geschwulstmasse verwachsenen Gland. sublingual. wurden mitentfernt. Vereinigung der Mucosa des Mundbodens durch fünf Nähte. Desinfection der grossen Wundhöhle mit Chlorzinklösung. Einlegen eines Drains in den unteren Wundwinkel, Doppelnaht. Lister'scher Verband. Herr Prof. Roth hatte die Freundlichkeit, die nähere Untersuchung der exstirpirten Muskelsţücke zu übernehmen und schreibt darüber, wie folgt:

"Die 8 in Müller'scher Flüssigkeit ubersandten Stticke, zusammen eine etwa hïhnereigrosse Masse bildend, aus Theilen des Mundbodens, etwas Schleimhaut und den 2 Gland. sublinguales, wesent- 
lich aber aus Musculatur (M. geniohyoidei und genioglossi) bestehend, welche sich nur an wenigen Stellen normal zerfasern lässt, bietet meist eine ziemlich derbe Beschaffenheit dar und enthält einige bis bohnengrosse, homogene, weissliche, speckige Einlagerungen. Diese letztern schwieligen Partien senden zwischen die besser erhaltene Musculatur lineäre und netzförmig verflochtene Auslänfer, so dass der Muskelquęrschnitt zum Theil regelmässig gefeldert erscheint. An einer der derbsten Partien ist der mediale Umfang der linken Gland. sublingual. ziemlich fest adhärent. Das mikroskopische Bild entspricht dem makroskopischen Befund vollständig. Sowohl Ihre als von mir aus verschiedenen Stellen gefertigte Schnitte lassen eine fibröse Wucherung erkennen, durch welche die Interstitien zwischen den Muskelfasern breiter erscheinen. Selbst an den derbsten speckigen Stellen fehlen die Muskelelemente nicht ganz, sind aber weit auseinander gedrängt, zum Theil körnig getrübt, ein einziges Mal wurde Vermehrung der Muskelkerne beobachtet. Das zwischen gelagerte Gewebe hat den Typus eines sclerotischen zellenarmen Bindegewebes, in welchem vielfach Haufen von kleinen, dichtgedrängten Rundzellen $(0,006-0,008 \mathrm{Mm}$.) eingestreut sind. Besonders scheinen diese Rundzellen dem Verlaufe kleiner Arterien zu folgen. Auch das Bindegewebe der linken Gland. sublingualis zeigt da, wo sie der sclerosirten Musculatur adbärirt, dieselbe kleinzellige Einlagerung.

Nirgend sarcomatöse oder carcinomatöse Structur, überall der Charakter einer chronischen interstitiellen Myositis.

Bei nochmaliger genauer Betrachtung der Fragmente ergab sich ein für die Entstehung, wie ich glaube, wichtiger Befund. Es findet sich nämlich auf der excidirten Schleimhaut, $9 \mathrm{Mm}$. nach hinten und aussen von der linken Caruncula sublingual. ein längliches, $5 \mathrm{Mm}$. langes und 1,5 Mm. breites Geschwür mit weicher, röthlicher Basis, von welchem ein 1-2 Mm. weiter, $5 \mathrm{Mm}$. langer mit weichem röthlichem Gewebe ausgekleideter Gang in die Tiefe fiihrt und bis in die schwieligen, am innern Rand der linken Gland. sublingual. gelegenen Partien hineinreicht. In diesem Gang liegen Eiter- und rothe Blutkörperchen; die Auskleidung desselben, wie auch das Gewebe der Geschwürbasis ist mit gefässreichen, zum Theil in Zerfall begriffenen Granulationen ausgekleidet. Der Duct. Wharton. passirt $2 \mathrm{Mm}$. lateralwärts von der Fistel und ist intact. Demnach scheint es mir kaum zweifelhaft, dass dieses Geschwïr und die daran anschliessende Myositis demselben Reize - etwa einem Fremdkörper? - ihren Ursprung verdanken ". 
Bezüglich des weiteren Krankheitsverlaufs war von Wichtigkeit, dass die Schleimhautuähte in Folge der Zungenbewegungen durchrissen und so die Mundhöhle mit dem Secret der Wundhöhle überschwemmt wurde. Eine erste Vereinigung wurde so verunmöglicht. Der Kranke bekam dann auch 8 Tage lang ein mässiges remittirendes Fieber und die Wunde eiterte ziemlich stark, was jedoch eine primäre Vereinigung der Ilautwunde bis auf das Drainloch nicht hinderte. Ein Theil der Schleimhant und der vorderste Theil der Zungenspitze fielen gangränös ab. Vom 17. Sept. an war der Kranke fieberfrei, die Wunde hatte sich gereinigt. Die Bewegungen der Zunge waren noch sehr mangelhaft und dem entsprechend war auch die Aussprache der Zungen- und Gaumenbuchstaben noch sehr mangellhaft. Die Zungenhartgaumenconsonanten D, T war der Kranke zu formen niclit im Stande, die Zungenweichgaumenconsonaten $\mathrm{K}, \mathrm{G}, \mathrm{R}$ waren undeutlich und die zusammengesetzten Consonanten $\mathrm{Z}$ und $\mathrm{X}$ konnten nicht ausgesprochen werden. Am 26. Sept. war die Wunde am Hals verlieilt, während unter der Zungenspitze noch eine kleine granulirende Wunde sich fand. Der vordere Theil der Zunge ist etwas kürzer und schmäler geworden, auch ist diese Partie ziemlich fixirt. Die Zungenspitze kanu zwar gehoben und gesenkt werden, aber die seitliche Beweglichkeit ist sehr gering. Während Patient früher beim Sprechen und Essen stechende Schmerzen vorn in der Zunge hatte, sind jetzt sümmtliche Bewegungen des Organs schmerzfrei. Die Sprache ist deutlicher geworden, beim Sprechen ist fast jedes Wort verständlich, während beim raschen Lesen Patient nur bei grosser Aufmerksamkeit verstanden werden kann.

Bis zum 2. October machte die Beweglichkeit der Zunge noch einige Fortschritte. Entlassung aus dem Spital am 4. October.

Am 23. October zeigte sich der Kranke wieder:

Er ist, wie immer, blülıend und gesund. Dic lineäre Narbe bildet einen dünnen Strang. Von einer Geschwulst unter dem Kiefer oder am Zungenboden ist nichts mehr wahrzunehmen. Der Zungenstumpf hat die frühere Grösse, der vorderste Theil, aus Narbensubstanz bestehend, ist etwas härter als die hintern Partien und entschieden nicht grösser, als bei der letzten Untersuchung. Die Beweglichkeit der Zunge hat nicht erheblich zugenommen. Sprechen und Schlingen sind ganz schmerzlos, ersteres noch etwas pappig und undeutlich. Einzelne Buchstaben kann er noch immer nicht aussprechen, z. B. sagt er statt $Z$, tscht.

Zuletzt sah ich den Kranken am 11. December und notirte, wie folgt:

Allgemeinbelinden ganz gut, Patient fühlt sich wohl und arbeitet den ganzen Tag. Aussehen blühend. Einmal zeigte sich eine leichte Schwellung unter dem rechten Kicferwinkel, die rasch wieder verschwand. Die Zunge erscheint gegen früher geschwunden, die Spitze ist am Mundboden fixirt, die rechte Hälfte etwas voluminöser und länger, als die linke, der vorderste friblher harte Theil ist weich geworden und unterscheidet sich in Bezug auf Consistenz nicht mehr von der übrigen Zunge. Die seitliche Beweglichkeit hat etwas zugenommen, geht aber um die fixirte Zungenspitze als Hypomochlion. Am Zungenboden so wenig, wie vom Halse aus sind noch harte Stellen zu fühlen, Narbe lineär, etwas hypertrophisch. Die Sprache ist wieder etwas deutlicher geworden, hat aber 
imper noch etwas Schwerfälliges. In Bezug auf die einzelnen Buchstaben ist folgendes erwähnenswerth: Von den Lippenbuchstaben kann

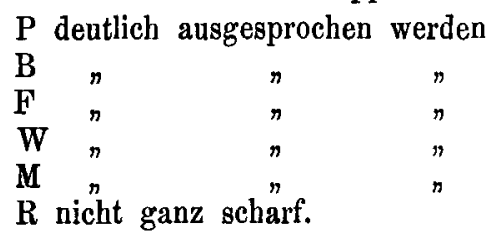

Von den Zungenbuchstaben werden nur N, R, L, Sch ganz scharf articulirt, statt $T$ sagt er Che, statt D Che, statt $S$ Esch, statt $T$ h Hehah.

Von den Gaumenbuchstaben werden deutlich ausgesprochen $\mathrm{K}, \mathrm{G}$, Ch H, statt Z Tscht.

Es lag also, wie aus der Untersuchung der exstirpirten Muskelstilcke resultirt, eine interstitielle sclerosirende Myositis einzelner Zungentheile vor. Eingeleitet wurde der entzundliche Process ohne Zweifel durch die Speichelsteine und unterhalten durch das von Herm Professor Roth gefundene kleine Geschwïr neben dem Duct. Whart., das wohl seinerseits ebenfalls den Speichelsteinen seine Entstehung verdankt. Uns ist dieses Geschwtir bei den wiederholten Untersuchungen der Mundhöhle entgangen, was um so erklärlicher ist, da der Kranke selbst von dem Vorhandensein einer wunden Stelle im Munde keine Ahnung hatte und bei der genauesten Palpation des Mundbodens niemals den geringsten Schmerz äusserte.

Die Entfernung der erkrankten Muskelpartien hatte ein verhältnissmässig glinstiges Resultat. Sie hat die Schlingbeschwerden beseitigt, die Sprache etwas gebessert und, wie aus der letzten Untersuchung des Kranken sicher hervorgeht, einen completen Stillstand der sclerosirenden Myositis zur Folge gehabt. Es bleibt meiner Ansicht nach zum mindesten zweifelhaft, ob eine exspectative oder eine lediglich auf das Geschwür gerichtete Behandlung das Gleiche oder Besseres erzielt hätte.

Für die Lehre von den Zungengeschwülsten ist es aber gewiss von grossem Interesse zu wissen, dass eine chronische Myositis Veranlassung zu einer am Halse prominirenden Geschwulst geben, und ich stehe nicht an auch jetzt zu wiederholen, was schon vor der Operation bemerkt wurde, einen malignen Tumor vortäuschen kann. In Zukunft allerdings wird das Vorhandensein von Speichelsteinen oder die Existenz eines Geschwürs am Mundboden diagnostische Anhaltspunkte geben können, aber bis jetzt war es durchaus unbekannt, dass Speichelsteine und ihre Folgen eine sclerosirende Myositis in Form eines abgrenzbaren Tumors veranlassen können. Das rasche Wachsthum des myotischen Herdes ist mir noch heute ein Räthsel, 
Sclerosir. Myositis d. Zungenwurzel, einen malignen Tumor vortăuschend. 375

da doch gewöhnlich die Entwicklung dieser Processe längere Zeit beansprucht, aber es ist sicher, da nicht anzunehmen ist, dass der intelligente Kranke von dem Vorhandensein einer Geschwulst unter der Zunge längere Zeit keine Kenntniss gehabt hätte.

Das Krankheitsbild, das aus dieser Beobachtung resultirt und, wie ich hoffe, bald durch ähnliche neue Fälle ergänzt und vervollständigt werden wird, stellt sich folgendermaassen dar:

Es kommt in der Zunge in Folge von Speichelsteinen und vielleicht anch anderer mechanischer Insulte eine sclerosirende Myositis vor, deren klinisches Substrat ein scheinbar abgrenzbarer, am Halse zum Vorschein kommender Tumor ist. Dieser Tumor wächst rasch, hat eine hart elastische, stellenweise knorpelharte Consistenz und glatte Oberfläche. Er ist bei starkem Druck schmerzhaft, erschwert das Sprechen, die Kau- und Schlingbewegungen, macht letztere schmerzhaft und kann consensuelle Schwellung benachbarter Lymphdrüsen im Gefolge haben. Unterscheidungsmerkmale zwischen einem von den Gebilden des Mundbodens ausgehenden malignen Tumor und dieser Muskelgeschwulst können unter Umständen Speichelsteine, geschwürige Processe an Mundboden etc. geben. Die Entfernung der schwieligen Massen bringt den chronisch-entzindlichen Process zum Stillstand und beseitigt einen Theil der dadurch bedingten Beschwerden. 